\title{
Interaction between cracking, delamination and buckling in brittle elastic thin films
}

\author{
W. P. Vellinga - M. Van den Bosch • \\ M. G. D. Geers
}

Received: 15 April 2008 / Accepted: 1 October 2008

(C) The Author(s) 2008. This article is published with open access at Springerlink.com

\begin{abstract}
A discrete lattice based model for the interaction of cracking, delamination and buckling of brittle elastic coatings is presented. The model is unique in its simultaneous incorporation of the coating and of disorder in the interface and material properties, leading to realistic 3D bending (and buckling) behavior. Results are compared to the literature. In the case of cracking, the key role of a stress transfer correlation length $\xi$ in establishing a scaling behavior for the brittle fracture of thin films is shown to extend to all geometrical and material properties involved. In the scaling regime of crack density in uniaxial tension cracking and delamination are found to occur simultaneously. In uniaxial tension of films with an internal biaxial compressive stress, the predicted initiation of buckles above delaminated areas near crack edges in the model is remarkably similar to experimental results.
\end{abstract}

Keywords Cracking - Delamination - Buckling · Lattice model

W. P. Vellinga ( $\varangle)$

Applied Physics, University of Groningen, Groningen,

The Netherlands

e-mail: w.p.vellinga@rug.nl

M. Van den Bosch · M. G. D. Geers

Mechanical Engineering, University of Technology

Eindhoven, Eindhoven, The Netherlands

\section{Introduction}

Coatings are used to protect surfaces in many applications and failure of a coating may lead to degradation of required engineering properties. Quite generally, coating failure is due to stresses that trigger irreversible mechanical deformation mechanisms, the most important of which are cracking, delamination and buckling. All of these mechanisms may appear in successive stages of deformation and interact with each other, as illustrated in Fig. 1.

The engineering interest in the field is focussed on preventing coating failure but the fascinating array of patterns that may form in these coatings (of which Fig. 1d shows an example) have attracted interest from various quarters.

This has resulted in a wide range of publications dealing with the field that can roughly be divided in two sets: firstly continuum mechanics literature dealing with crack propagation and crack patterns near bi-material interfaces (e.g. Xia and Hutchinson 2000; Hutchinson and Suo 1992; Beuth 1992; He et al. 1998; Gille 1984; Mezin et al. 1989a) and secondly literature that discusses so-called "lattice models" in which coating, substrate and interface are represented by a lattice of points connected by bonds, springs or beams that can break irreversibly. Such lattice models have been studied in a wide variety of contexts that will not be discussed here; comprehensive reviews can be found in Ostoja-Starzewski (2002), van Mier (2007) and Alava et al. (2006). Initial work on the use of such 
Fig. 1 Illustration of deformation and failure mechanisms in supported brittle films under uniaxial tension. a-c Sketches of successive stages in the failure showing plane sections parallel (a1-c1) and perpendicular to the tensile load (a2-c2). Experimental image of stage $\mathbf{c}$ showing triangular buckles above delaminated areas between cracks. (The system consists of amorphous hydrogenated carbon deposited on $\mathrm{Al}$ substrate) Symbols for tensile direction (arrows) and plane cuts (coded lines) allow comparison of $\mathbf{a}-\mathbf{c}$ with d
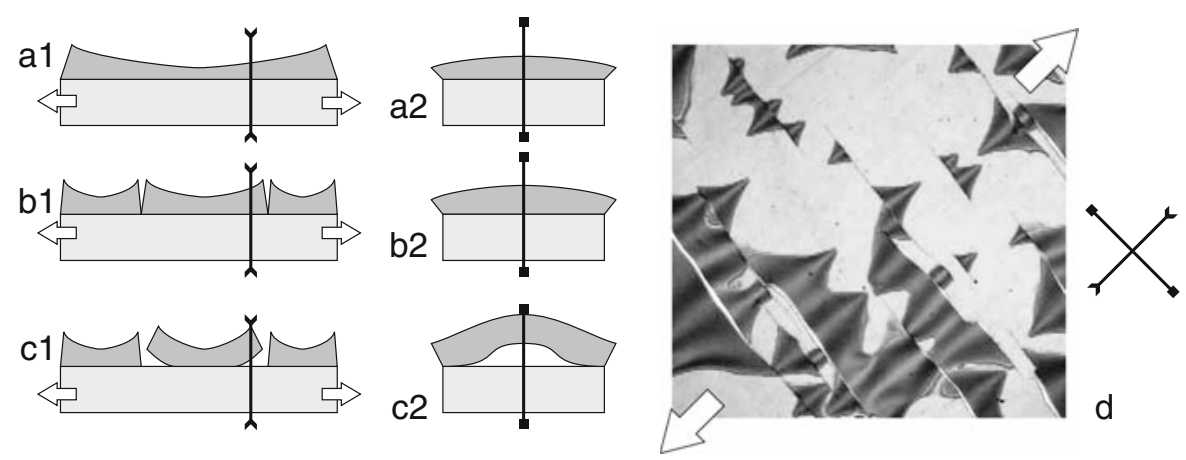

models for coatings was performed by Meakin (1987), Skjeltorp and Meakin (1988). Several adaptations anticipated in the papers by Meakin have been investigated, including models with viscoelastic Handge et al. (1997) and plastic coating elements Handge et al. (2001). Work has also been directed to the interaction between disorder and elastic properties Handge et al. (1999), pattern formation Leung and Neda (2000) and the (onset of) scaling regimes in patterns Hornig et al. (1996), Handge et al. (2000a). Part of the work has dealt with essentially 1D cracking systems in which the scaling properties may be analytically approximated, while still being relevant to experimental (or testing) situations Morgenstern et al. (1993), Handge et al. (1999), Handge et al. (2000b).

A number of other papers discussed systems in which the interface is allowed to deform irreversibly during the simulation, e.g. by slippage Kitsuzenaki (1999).

Buckling, which requires a realistic description of the bending stiffness of the coatings has been studied on relatively few occasions, most notably by Jagla (2007) who discusses the interaction of delamination and buckling in layers with a compressive internal stress.

This paper aims to treat the most general situation in which all three deformation mechanisms mentioned above, cracking, delamination and buckling are allowed to interact freely. Disorder in the material properties can also be taken into account. Some key characteristics of the behavior of a specific system that shows this type of interaction are evident from Fig. 1d showing deformation phenomena of a brittle elastic amorphous carbon layer supported by polished Al. In response to uniaxial tension (indicated by the arrows), cracks develop perpendicular to the tensile direction. Near the crack edges triangular delaminated areas are visible above which the coating has buckled. From the location of the buckles it is clear that the three deformation mechanisms interact in this case. Figure 1a-c shows sketches of the successive deformation stages involved.

The paper is organized as follows: the numerical model and its features are introduced in Sect.2. In Sect. 3, results of cracking are presented. The idea is to establish the validity of the approach by a comparison with the literature, and show some original results on scaling behavior. Compared to what is commonly used in the literature, a slightly different way of presenting the scaling relations for the cracking of thin films will be used. In Sect. 4 results of simultaneous cracking and delamination are discussed. Finally the results of cracking, delamination and buckling are given in Sect. 5 .

\section{Lattice based coating-substrate model}

The lattice model consists of three regions: the substrate, the interface and the coating, for which the characteristic unit cells are shown in Fig. 2.

Different boundary conditions may be applied as shown in Fig. 3. In case of a 2D model periodic 


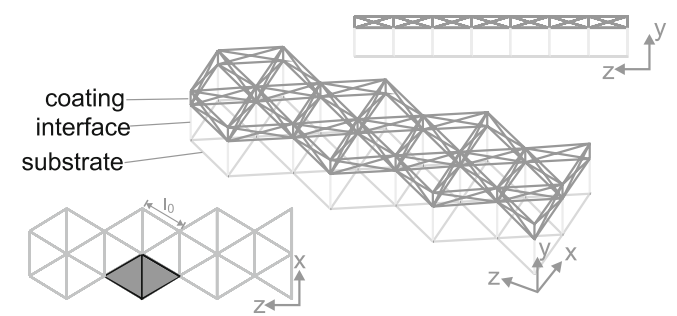

Fig. 2 A $2 \times 73 \mathrm{D}$ model. In the side- and perspective-view black represents the coating, light gray the interface and dark gray the substrate. In the top view the black lines and grey area indicate the projection of a single unit cell. The length of the interface is magnified in the figure for viewing purposes

boundary conditions (PBC's) are applied on a model only one unit wide in $\mathrm{X}$-direction, see Fig. 2. This geometry is a representation of an array of straight cracks, such as shown in Fig. 3. In such a model a crack cannot propagate and delamination and buckling may initiate and propagate only along the interface in z-direction. Evidently, PBC's can also be applied to models with a width of several units, but these are not discussed here. In the 3D models cracks may propagate, and delamination and buckling behavior may develop.

Equilibrium configurations are determined in a standard fashion with the Finite Element Method (FEM). Two different types of elements are used. Firstly, in the coating and the substrate: brittle linear elastic spring elements. These elements do not have a bending stiffness and instead a 3D network in the coating provides the bending stiffness necessary for the simulation of thickness effects in delamination and buckling. Secondly, in the interface: brittle linear elastic leaf-springs that transfer loads perpendicular as well as parallel to their axis.

Elements in the coating are considered to break at predetermined fracture forces (equivalent to predetermined elongations), which represents cracking. If the force in a spring element $n$ exceeds its assigned fracture limit $F_{n}^{b}$, the element is removed from the model.
The leaf spring elements in the interface are also considered to break when their elongation equals a predetermined value, which is equal to $F_{i}^{b} / k_{i, a x}$. Perpendicular to the leaf-spring axis a force $F_{p}$ leads to an associated displacement $F_{p} / k_{i, p}$, which causes a small elongation of the spring. Defining an angle $\theta$ with $\tan (\theta)=\left(F_{p} / h_{i} k_{i, p}\right)$ and $h_{i}$ the leaf spring length one finds for the axial elongation necessary to cause fracture (first order approximation, for small values of $\theta) F^{b} / k_{i, a x}-h_{i} \tan (\theta)^{2} / 2$. This effectively leads to a type of mixed-mode delamination criterion in which the energy necessary for delamination (i.e. removal of the element) is different in pure axial loading (in this model coinciding with mode I fracture) than it is in pure perpendicular (mode II) loading. This is illustrated in Fig. 4 where the mode I and mode II energies associated with breaking are plotted. What is plotted is the fracture energy in the two modes normalized with respect to the pure mode I value. In this paper $k_{a}=k_{p}=k_{i}$. Results will depend on the ratio $k_{a} / k_{p}$ (e.g. reducing $k_{p}$ reduces the mode II contribution to the energy release rate) but this is not addressed in this paper.

The breaking force $F_{n}^{b}$ is defined as:

$F_{n}^{b}=F^{b}+W \cdot F_{a m p}^{b}$ with $\quad(-1<W<1)$

where $F^{b}$ is the mean breaking force (of the entire coating), $F_{a m p}^{b}$ is the amplitude of the scatter around $F^{b}$ and $W$ is taken randomly from a uniform distribution.

Disorder parameters $\kappa$ are defined as:

$\kappa_{c}=\frac{F_{c, a m p}^{b}}{F_{c}^{b}}$ and $\kappa_{i}=\frac{F_{i, a m p}^{b}}{F_{i}^{b}}$

where the subscript $\left(_{c}\right)$ indicates the coating and $\left(_{i}\right)$ the interface.

The geometry of a coating lattice is expressed as follows: a $n \times m$ 3D model has $n$ units of length $l_{0}$ in $\mathrm{x}$-direction (in the plane $z=z_{\min }=0$ in Fig. 2) with a total length $L_{0 x}=n l_{0}$ and it has $m$ units in z-direction with a total length $L_{0 z}=\frac{1}{2} \sqrt{3} m l_{0}$. For 2D models discussed here $n \equiv 1$ so only $m$ is relevant and the initial length $L_{0} \equiv L_{0 z}$.

Fig. 3 Static properties of 3D straight cracks and delamination (a) can be captured in a periodic $2 \mathrm{D}$ model (b). Crack propagation along arbitrary directions, delamination and buckling can only be captured in a 3D model (c)

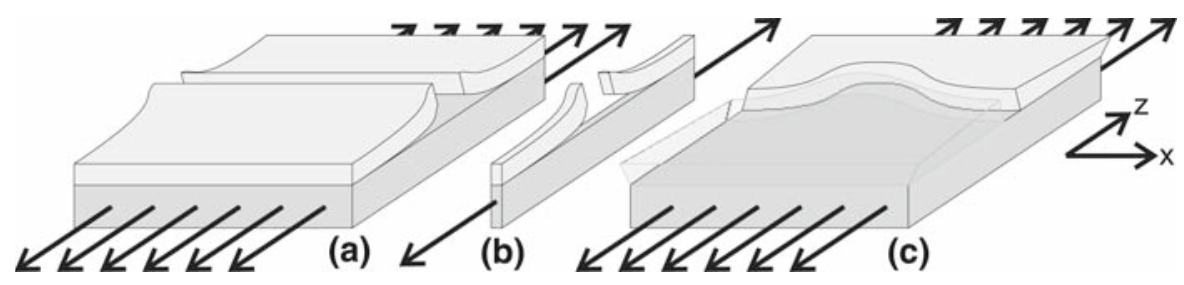




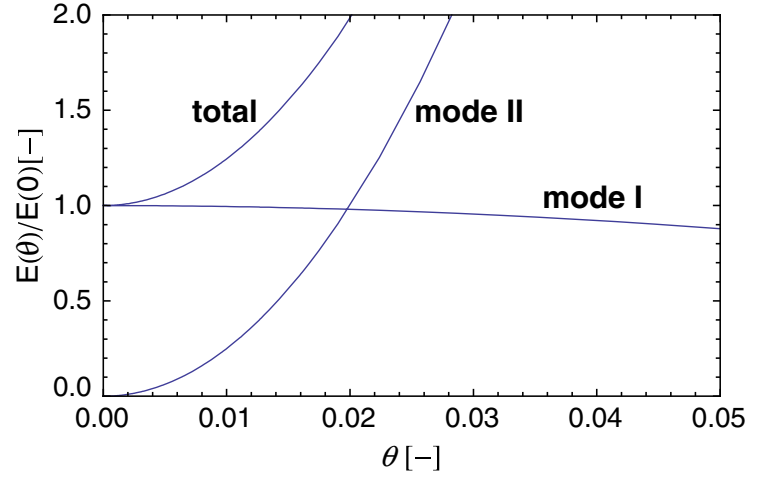

Fig. 4 Energy stored in spring at fracture as a function of angle $\theta$ (see text). For $\theta=0$ the fracture is equivalent to pure axial loading or pure mode I. All values have been normalized with respect to that value. The figure shows the mode I component, the mode II component and the total fracture energy. The ratio $k_{a} / k_{p}$ is equal to 1 here

All nodes are initially regularly ordered such that equilateral triangles are created in the xz-plane. Geometrical disorder is introduced by displacing nodes across a distance $d r$ in direction $d \alpha$ in the xz-plane, according to:

$d r \in W_{2} \cdot d r_{\max }$ and $d \alpha \in W_{3} \cdot 2 \pi$

where $W_{2}$ and $W_{3}$ are distributions (in this case: uniform) with $0 \leq W_{i} \leq 1$ and $\mathrm{i}=2,3$. The maximum displacement of a node, $d r_{\max }$, can be defined to control the amount of geometrical disorder $\kappa_{g}$ :

$\kappa_{g}=\frac{d r_{\max }}{\frac{1}{2} l_{0}}$

where $l_{0}$ is the initial element length without disorder in the model.

\subsection{Solution}

The simulation is load-controlled and the nodal displacements are calculated in each loading step using an incremental solution algorithm based on a piece-wise standard application of linear FEM:

$\underline{u}=\underline{K}^{-1} \underline{f}$

Here $f$ is the nodal force column, $\underline{K}$ the stiffness matrix and $\underline{u}$ the column with the nodal displacements. A preconditioned conjugate gradient method (PCG) is used to solve the system. As preconditioner the incomplete Cholesky factorization (ICF) of the stiffness matrix is used. In every increment the external force is changed in such a way as to precisely fracture one element, thus making optimal use of the linear relation between the applied forces and the nodal displacements (Eq. 5). However, forces perpendicular to element axes induce a slight non-linearity, which is taken into account. For the determination of the required load step the lengths of the springs are updated to assess this property. When the next weakest element is known, it is broken. To improve on the efficiency, elements are broken in sets. When the model structure is projected along the y-axis certain sets of elements fall on top of each other, these sets are called "groups". Similarly, a projection along the x-axis permits to identify sets, which are called "families". A "family" consists of a number of "groups". In the model we choose to always break either the group (3D) or the family (2D) to which the weakest element belongs. Since a group is the smallest part of the coating that can crack, the cracks cannot be bridged along the ydirection. The coating is cracked from the top-surface right down to the interface. If the elements do not experience large strains or rotations $\underline{K}$ is not entirely recalculated. Rather, at each loading step the affected fraction of $\underline{K}$ is updated to incorporate the element removal. This significantly decreases the required calculation time for every increment. Likewise, the ICF is not recalculated every increment, but only when the time spent in recalculating outweighs the extra time spent in iterating caused by bad preconditioning.

\subsubsection{Residual stresses}

Residual stresses are directly assigned to individual elements, yielding an extra contribution to the internal force of these elements.

The determination of the nodal displacements (e.g. when determining the weakest element) in the presence of residual stresses is performed as follows. First, the displacements $\underline{u}_{i n t}$ caused by only the internal forces are determined. Then, the displacements $\underline{u}_{\text {ext } 1 N}$ caused by an external unit load of $1[N]$ are calculated. The real displacements $\underline{u}_{i}$ caused by an external force $F_{\text {ext }}$ and all internal forces are now given by:

$\underline{u}_{i}=\underline{u}_{e x t 1 N} F_{\text {ext }}+\underline{u}_{i n t}$

The actual load $F_{\text {ext }}$ is then determined through the weakest link, i.e. the element which first 'hits' its fracture limit. 


\subsubsection{Buckling}

The simulation of large displacements in buckling is performed in a incrementally step-wise linear fashion as well. The method discussed previously is again adopted but the stiffness matrix $\underline{K}$ is updated at the end of each increment. This has the desired influence on the behavior of the model provided adequately small steps are taken. Prior to updating $\underline{K}$, the model must be in equilibrium, which requires an extra solver step.

\section{Results: in-plane cracking of the film}

\subsection{Correlation length}

It is possible to establish a close correspondence between the model parameters used in the lattice model and material parameters representative for experimental situations. Moreover a set of simplifying assumptions known as the shear-lag approximation provide interesting insights in the interplay between material parameters (including disorder) and geometry when cracking occurs. In a 2D representation as shown in Fig. 5, a coating $c$ is connected to the substrate $s$ by the interface $i$. The substrate is subjected to a uni-axial uniform stress $\sigma_{0}$ in $x$-direction. The following stressstrain relation holds for both the coating and the substrate:

$\varepsilon_{x x}=\frac{\sigma_{x x}}{E}$.

The shear-lag approximation relates the shear stress $\tau_{i}(x)$ in the interface to the stress in the coating and the stress change $\Delta \sigma_{s}$ in the substrate:

$\tau_{i}(x)=h_{c} \frac{\partial \sigma_{c}}{\partial x}=-h_{s} \frac{\partial \Delta \sigma_{s}}{\partial x}$

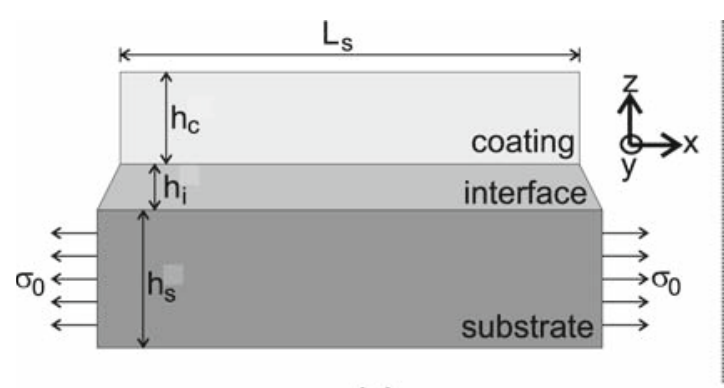

(a)

From Eq. 8 follows:

$\sigma_{c}(x)=\frac{1}{h_{c}} \int_{0}^{x} \tau_{i}(x) d x$

and

$\sigma_{s}(x)=\sigma_{0}-\frac{1}{h_{s}} \int_{0}^{x} \tau_{i}(x) d x$

At this point, a stress-strain relation for the shear stress at the interface has to be specified. Using a linear elastic shear approximation

$\tau_{i} \approx \frac{C}{w}\left(u_{c}-u_{s}\right)$,

with $C$ a shear modulus and $w$ a characteristic interfacial distance across which the displacement occurs. In relation with the adopted lattice model we take $C=G_{i}$ and $w=h_{i}$. Using this result in Eq. 8 yields:

$\frac{\partial^{2} \sigma_{c}}{\partial x^{2}}=\frac{G_{i}}{h_{c}}\left(\frac{\varepsilon_{c}-\varepsilon_{s}}{h_{i}}\right)$

From Eq. 8, a relation between $\varepsilon_{c}$ and $\varepsilon_{s}$ follows that can be used to arrive at:

$\frac{\partial^{2} \sigma_{c}}{\partial x^{2}}-k_{1}^{2} \sigma_{c}(x)=-k_{2}$

with:

$k_{1}^{2}=\frac{G_{i}}{h_{i}}\left(\frac{1}{h_{c} E_{c}}+\frac{1}{h_{s} E_{s}}\right)$ and $k_{2}=\frac{G_{i}}{h_{c} h_{i} E_{s}} \sigma_{0}$

Using the boundary conditions $\sigma_{c}\left(-L_{s} / 2\right)=$ $\sigma_{c}\left(L_{s} / 2\right)=0$ gives:

$\sigma_{c}(x)=-\frac{k_{2}}{k_{1}^{2}}\left(1-\frac{\cosh \left(k_{1} x\right)}{\cosh \left(\frac{k_{1} L_{s}}{2}\right)}\right)$

and

$\tau_{i}(x)=h_{c} \frac{k_{2}}{k_{1}} \frac{\sinh \left(k_{1} x\right)}{\cosh \left(\frac{k_{1} L_{s}}{2}\right)}$

Fig. 5 a Substrate under a uni-axial load. $\mathbf{b}$ The stress components in a part of the substrate, interface and coating

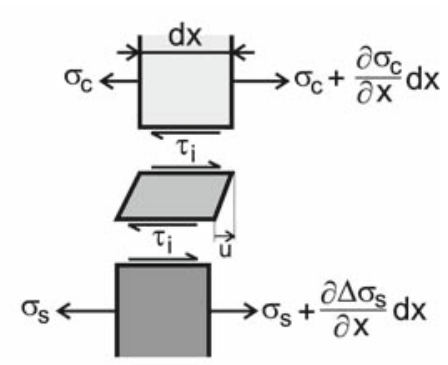

(b) 


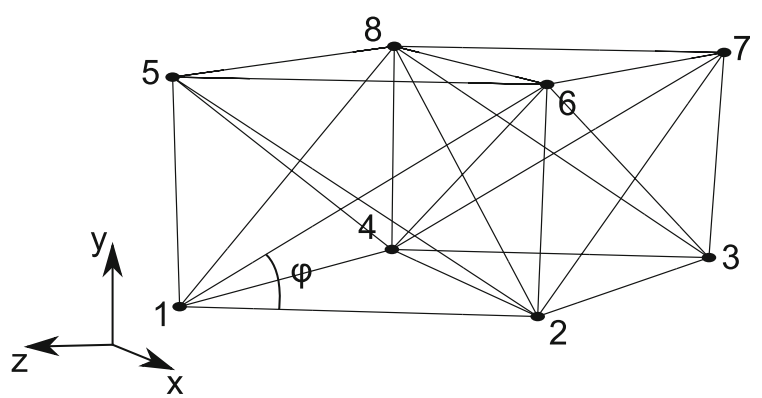

Fig. 6 Illustration of unit cell used in calculation of representative stiffness tensor for the coating

Clearly, a correlation length $k_{1}^{-1}=\xi$ can be introduced:

$\xi=\left(\frac{G_{i}}{h_{i}}\left(\frac{1}{h_{c} E_{c}}+\frac{1}{h_{s} E_{s}}\right)\right)^{-\frac{1}{2}}$

In the adopted lattice model, spring elements are used and we define $C_{s}, C_{i}$ and $C_{c}$ as the spring constants of the substrate, interface and coating elements respectively. Of course it is desirable to establish equivalent continuum moduli from the lattice model parameters.

For the coating a well-established homogenisation procedure has been used Kouznetsova et al. (2002) to determine the components of the representative stiffness tensor. In this procedure, periodic boundary conditions are assumed for unit cells see Fig. 6. Introducing the planar $(\mathrm{x}, \mathrm{z})$ coordinates of the corners as $x_{i}$ and the non-planar y coordinates as $y_{i}$, with corners in the lower plane numbered from 1 to 4 counterclockwise and in the upper plane numbered from 5 to 8 counterclockwise, the periodic boundary conditions enforced on the unit cell are: $x_{1}-x_{2}=x_{4}-x_{3}, x_{5}-x_{6}=x_{8}-x_{7}$, $y_{1}=y_{2}=y_{3}=y_{4}$ and $y_{5}=y_{6}=y_{7}=y_{8}$. Using these conditions it is found that the coating, as described by the spring model is a transversely isotropic medium with the following non-zero tensor components:

$c_{11}=c_{22}=\frac{3 \sqrt{3} C_{c}(3+\cos 2 \phi)}{4 h_{c}}$

$c_{33}=\frac{2 h_{c}\left(C_{c}+6 C_{c}(\sin \phi)^{2}\right)}{\sqrt{3} l_{0}^{2}}$

$c_{55}=c_{66}=\frac{2 \sqrt{3} C_{c} h_{c}(\cos \phi)^{2}}{\sqrt{3} l_{0}^{2}}$

$c_{12}=\frac{\sqrt{3} C_{c}(3+\cos 2 \phi)}{4 h_{c}}$ $c_{13}=\frac{\sqrt{3} C_{c} \sin 2 \phi}{l_{0}}$

This characterises the elastic properties of the layer completely. Expressions for the Young's moduli are not quite as simple, since the Poissons's ratio's also depend on the height $h_{c}$ and the angle $\phi$. However this dependence is rather weak. This effect can be captured by a term $c(\phi)$ that varies in value between roughly 0.8 and 0.9 if $\phi$ varies from 0 to $\frac{\pi}{2}$. So for the in-plane Young's modulus of the film we can use

$E_{c}^{m}=c(\phi) \frac{3 \sqrt{3} C_{c}(3+\cos 2 \phi)}{4 h_{c}}$.

Here, the superscript $m$ indicates model properties. For thickness changes in a regime of small angles $\phi$ the cosine term is also more or less constant and the in-plane Young's modulus depends to a good approximation linearly on the spring constant and the inverse coating height $\frac{1}{h_{c}}$. To further establish correspondence between model parameters, and (experimental) parameters that appear in the shear-lag formulation, one can introduce the following quantities:

$G_{i}^{m}=\frac{4 C_{i} h_{i}}{\sqrt{3} l_{0}^{2}}, \quad E_{s}^{m}=\frac{\sqrt{3} C_{s}}{4 h_{s}}$.

$h_{i}$ is the interface height in the model. $h_{s}$ serves only to scale the spring constant $C_{s}$. Using these equivalences it is possible to relate force profiles in coating and interface or the correlation length in the model to those calculated in Eqs. 15, 16 and 17.

\subsection{Scaling behavior}

In Fig. 7, the force profile $F_{c}\left(x / L_{s}\right) / F^{b}$ in a part of a coating is plotted for different values of $L_{s} \xi^{-1}$, with $L_{s}$ the (mean) segment length. The set of curves is representative for profiles occurring in a coating that is fractured increasingly. When $L_{s} \xi^{-1} \gg 1$ the stress in the coating reaches the maximum value in a "plateau" region. When $L_{s} \xi^{-1} \ll 1$, the stress cannot reach this plateau value, and the stress profile instead has a parabolic shape.

In uniaxial tensile experiments the behavior of the mean segment length $L_{S}$ as a function of the applied strain $\varepsilon$ is known to show two distinct regimes. This is a direct consequence of the behaviour of the maximum segment stress $F_{c}^{\max }\left(L_{s}(\varepsilon)\right)$. Experimental work directed towards elucidating patterning and scaling behavior 


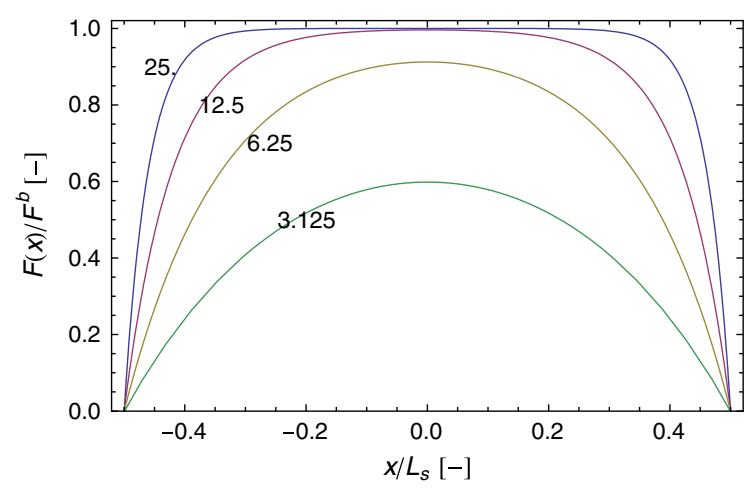

Fig. 7 Force profiles at constant external force, showing the influence of decreasing $L_{s} \xi^{-1}$

in crack patterns was reported in e.g. Gille and Wetzig (1983), Mezin et al. (1989b), Yanaka et al. (1998, 1999), Walmann et al. (1996), Leung et al. (2001), Handge et al. (1999).

The first regime is characterized by a rapidly increasing density of cracks without a further increase of the external force (or strain). In a displacement controlled tensile test many cracks would suddenly appear in this first regime. In the second regime, an increasing macroscopic strain is needed before more cracks will initiate. Moreover in this mode a scaling regime develops. The onset of the scaling regime provides information on the correlation length, and the slope in the scaling regime is related to characteristics of the disorder in ("quality of") the coating Handge et al. (2000a), Morgenstern et al. (1993), Handge et al. (2000b). In the following the obtained segment length as a function of the overall strain $\varepsilon, L_{s}(\varepsilon)$ for our model system is illustrated. The aim is to show that it reproduces results from literature, and suggests that $L_{S}(\varepsilon)$ may be further exploited in the assessment of coating and interface properties.

The mean segment length $L_{s}$ has been defined as

$L_{s}=\frac{L_{0}}{n_{c r}+1}$ where $L_{0}$ is the initial model length and $n_{c r}$ is the number of cracks in the coating.

It is clear from the above that $L_{s}$ should be normalized with $\xi$. Moreover it seems reasonable to expect that $\varepsilon$ scales with $\varepsilon_{\max }^{b}$, so we introduce $\varepsilon^{n}$ :

$\varepsilon^{n}=\frac{\varepsilon}{\varepsilon_{\max }^{b}}$

The maximum breaking strain is given by:

$\varepsilon_{\max }^{b}=\frac{6 F^{b}}{E_{c}^{m} b h_{c}}$

where $F^{b}=F_{c}^{b}\left(1+\kappa_{c}\right)$ :

Results from a number of simulation run at different parameter values, shown in Table 1 are plotted as $L_{s} \xi^{-1}\left(\varepsilon^{n}\right)$ in Fig. 8. All results, regardless of the material and geometry parameters, are positioned on a single master curve, shown as a thick black line. The master curve is determined as follows: the maximum force in a segment as a function of the segment length is given by:

$F_{0, L_{s}}=k_{2} \xi^{2} b h_{c}\left(1-\operatorname{sech}\left(\frac{L_{s}}{2 \xi}\right)\right)$

For a break to occur at the centre of a segment this maximum force has to be equal to the breaking force, i.e. $F_{0, L_{s}}=F^{b}$, and thus $\varepsilon^{n}\left(L_{s}\right)$ of the mastercurve equals:

$\varepsilon^{n}\left(L_{s}\right)=\left(1-\operatorname{sech}\left(\frac{L_{s}}{2 \xi}\right)\right)^{-1}$

The particular scaling presented in Fig. 8 is derived for $\kappa_{c}=0$. In the case where disorder occurs, the behavior is known to deviate, as shown by Handge et al. (2001). The model description shown here reproduces their finding that the slopes of the curves at small values of $L_{S} \xi^{-1}$ change from $-\frac{1}{2}$ for $\kappa_{c}=0.0$ to $-\frac{1}{3}$ for $\kappa_{c}=1.0$, but here this is not further discussed.

Table 1 1 15012 D simulations

\begin{tabular}{llll}
\hline Simulation & Varied parameter & Simulation & Varied parameter \\
\hline 1 & None & 5 & $E_{s}->10 E_{s}$ \\
2 & $E_{i}->0.01 E_{i}$ & 6 & $F_{c}^{b}->2 F_{c}^{b}$ \\
3 & $G_{i}->10 G_{i}$ & 7 & $h_{i}->0.1 h_{i}$ \\
4 & $E_{c}->0.1 E_{c}$ & 8 & $l_{0}->0.5 l_{0}$ \\
\hline
\end{tabular}

Changes to parameters for curves depicted in Fig. 8. No disorder 


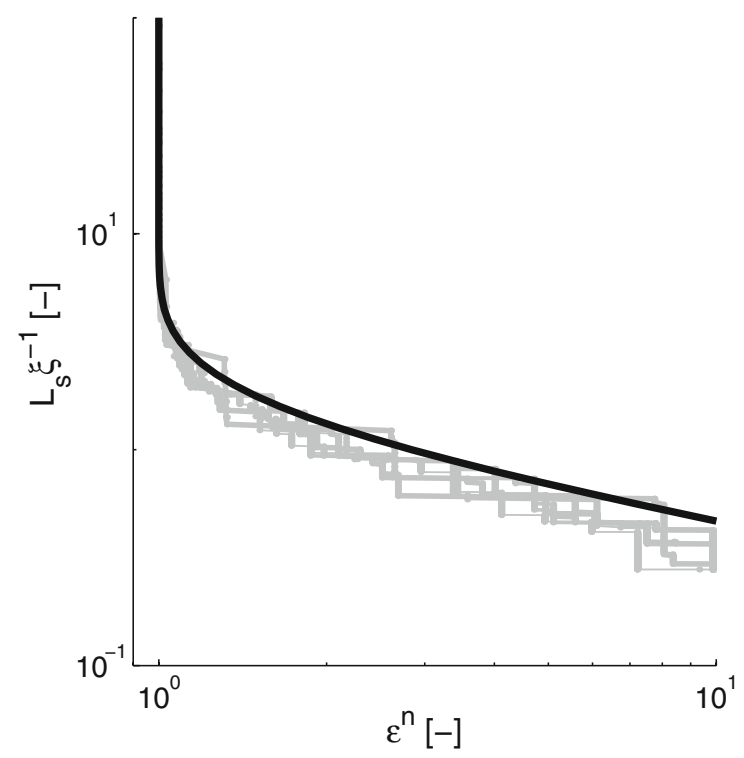

Fig. 8 Results of calculations specified in Table 1 plotted as $L_{s} \xi^{-1}\left(\varepsilon^{n}\right)$ together with master curve (see text)

\subsection{Crack initiation and propagation versus $\xi$}

The correlation length $\xi$ plays a similar role in 3D uniaxially loaded models as in 2D models, but additionally it also influences the propagation of cracks. This follows from the fact that a stress concentration appears in front of every crack, the amplitude of which depends on $\xi$.

Figure 9 shows crack patterns resulting after 2500 fracture events, for situations with different values of $\xi . L_{0} \xi^{-1}$ equals 64 (left), 128 (middle) and 256, (right). Note the clear difference in the crack patterns. Figure 10 shows $F_{\text {ext }}$ as a function of the number of fractured elements for sim1 and sim3.

The curve of sim1 shows characteristic spikes. The spikes represent the value of $F_{\text {ext }}$ necessary to initiate a crack in sim1. Due to the stress concentration at the

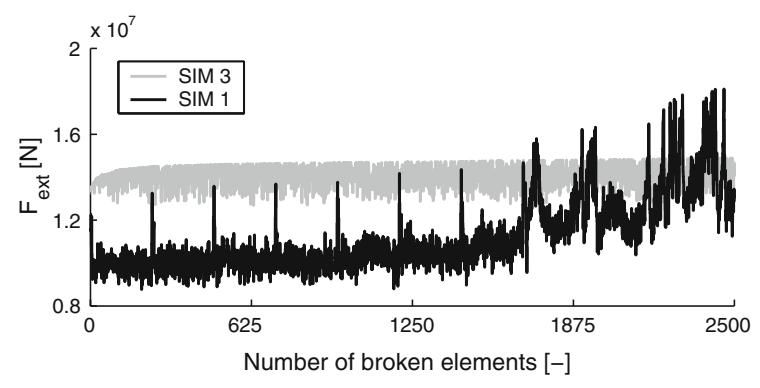

Fig. $10 F_{\text {ext }}$ as function of the number of broken elements for sim1 and sim3

crack tip, the force necessary to propagate the crack is significantly smaller than the nucleation force in this case. In displacement controlled experiments this would be observed as a crack that initiates and immediately propagates through the whole sample width. In sim1 after seven of such cracks (that appear as 7 spikes in Fig. 10), $L_{s}$ has decreased and becomes the lengthscale relevant for the stress concentration at the crack tip. Furthermore the fracture of the coating transfers from the regime where $\left(L_{s} \gg \xi\right)$ to a power-law scaling regime as discussed in 3.2. In the scaling regime, a steady increase in the external loading is needed to ensure further cracking. However, it can also be seen that the difference between the initiation and propagation forces becomes less pronounced. The $F_{\text {ext }}$ curve of sim3 in Fig. 10 does not present large spikes, because the stress transfer to the crack tip is much less efficient for small values of $\xi$. In this case the probability of nucleation of a new crack compared to the propagation of an already existing crack has increased.

\subsubsection{Crack propagation and disorder}

The influence of material disorder $\kappa_{c}$ and geometric disorder $\kappa_{g}$ on the crack initiation and propagation is shown in Fig. 11a-f. In Fig. 11a there is no material
Fig. 9 Influence of $\xi$ on the crack pattern after 2500 fractured elements in a $120 \times 1203$ D model. Tensile direction is vertical. From left to right $L_{0} \xi^{-1}=64,128$ and 256 . Cracks are black. $\kappa_{c}=0.1$ and $\kappa_{g}=0.1$. Greyscale indicates spring forces from zero, (black), to $F_{c}^{b}$, (white)

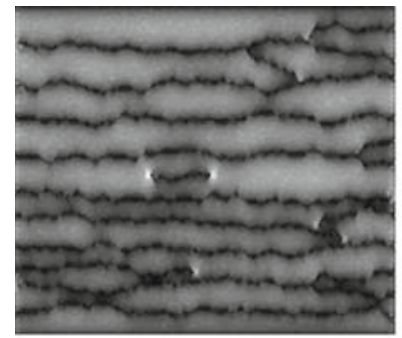

sim 1

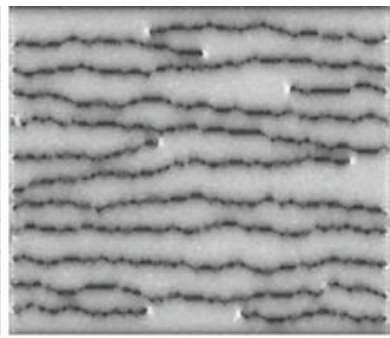

$\operatorname{sim} 2$

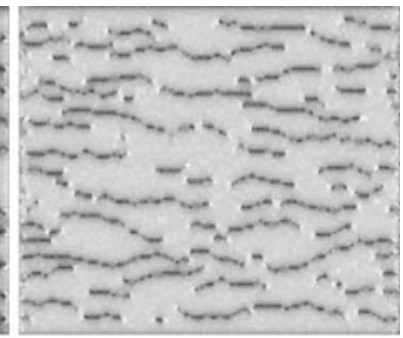

$\operatorname{sim} 3$ 
Fig. 11 3D uni-axial simulations with different values for $\kappa_{c}$ and $\kappa_{g}$. a $\kappa_{c}=0.0, \kappa_{g}=0.0$, b $\kappa_{c}=0.5, \kappa_{g}=0.0$, c $\kappa_{c}=1.0, \kappa_{g}=0.0$, d $\kappa_{c}=0.0, \kappa_{g}=0.0$, e $\kappa_{c}=0.0, \kappa_{g}=0.5$, f $\kappa_{c}=0.0, \kappa_{g}=1.0$. $L_{0} \xi^{-1}=9.9$. The tensile force is applied in vertical direction. All graphs are taken after the same amount of broken elements
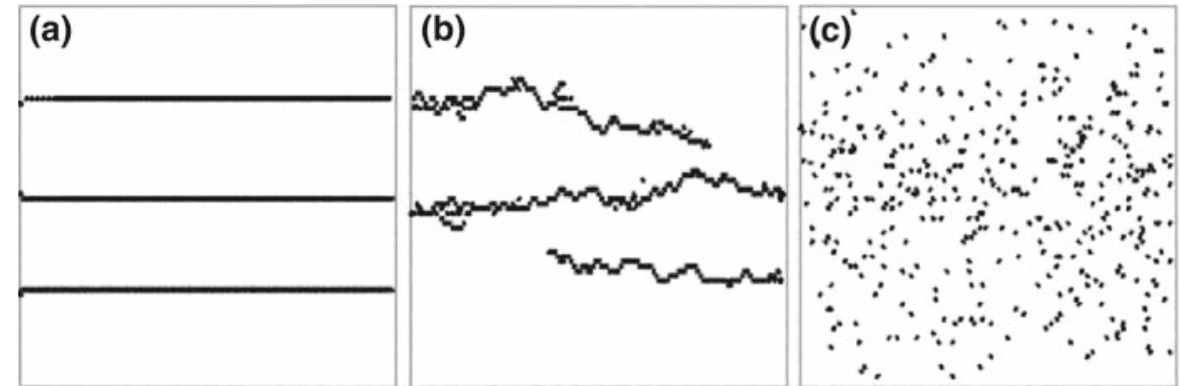

(d)

(e)

(f)
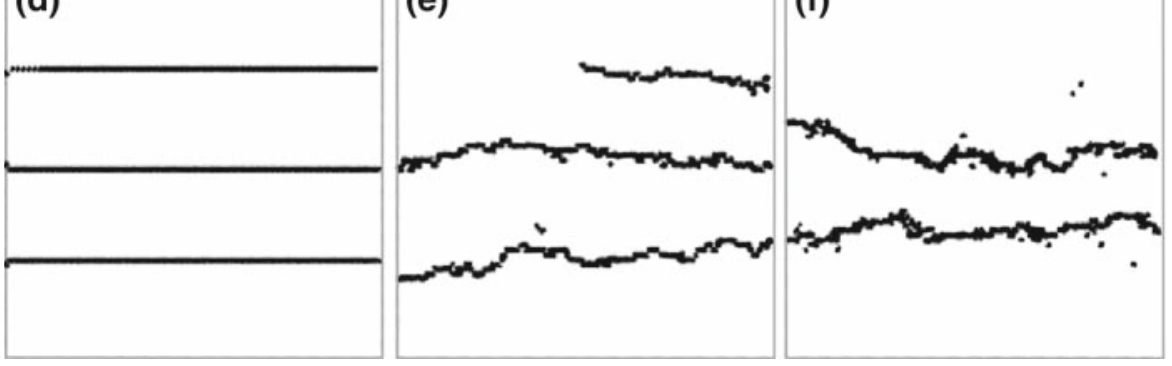

disorder, $\kappa_{c}=0$, and therefore the first crack will initiate in the middle since the forces are the highest there.

In Fig. $11 \mathrm{~b} \kappa_{c}=0.5$, which is large enough to cause fracture of elements that are not loaded with the highest force. This yields a crack that is not completely perpendicular to the tensile direction. In $11 \mathrm{c} \kappa_{c}=1.0$ and elements may fracture at a force of (nearly) zero value, through which propagation becomes a non-issue. The broken elements are not randomly located since the stress and the probability that an element will break are both highest in the middle. The influence of $\kappa_{g}$ is not as remarkable as that of $\kappa_{c}$. This is shown in Fig. 11d-f.

\section{Film cracking and delamination}

In this section we will investigate the interaction between cracking and delamination. In the presence of delamination nearby a crack the load transfer to the coating is partially inhibited, which may clearly influence further fracture events. In a typical sequence of uniaxial tension experiments it has been observed that after an initial increase in crack density, pockets of delaminated interface start to appear at the crack edges. Subsequently, cracking and delamination may occur side-by-side during the remainder of the experiment. This sequence of events indicates that the occurrence of cracks may also influence subsequent delamination. In this section the interaction is studied within the limits of the present model, but again departing from an essentially scalar shear lag approximation.

\subsection{Critical length for delamination}

First, two new quantities are defined: the normalized breaking strengths of the coating and interface: $\alpha_{c}$ and $\alpha_{i}$,

$$
\begin{aligned}
\alpha_{c}\left(L_{s}\right) & =\frac{F_{c, \max }\left(L_{s}\right)}{F_{c}^{b}} \text { and } \alpha_{i}\left(L_{s}\right) \\
& =\frac{F_{i, \max }\left(L_{s}\right)}{F_{i}^{b}}
\end{aligned}
$$

Where $F_{c, \max }\left(L_{s}\right)$ and $F_{i, \max }\left(L_{S}\right)$ are the maximum forces in the coating and interface as function of the segment length $L_{s} . F_{c}^{b}$ and $F_{i}^{b}$ are the breaking forces of the coating and interface respectively $\left(\kappa_{c}=\kappa_{i}=0\right)$. Using Eqs. 15 and 16 for the force profile in the coating and interface respectively, the maximum force in the coating $F_{c, \text { max }}$ and interface $F_{i, \max }$ may be expressed as:

$$
\begin{aligned}
F_{c, \max }\left(L_{s}\right) & =f_{1}\left(1-\operatorname{sech}\left(\frac{L_{s}}{2 \xi}\right)\right) \text { with }: f_{1} \\
& =k_{2} \xi^{2} b h_{c}
\end{aligned}
$$

and:

$F_{i, \max }\left(L_{s}\right)=f_{2} \tanh \left(\frac{L_{s}}{2 \xi}\right)$ with $: f_{2}=k_{2} \xi h_{i} b l_{0}$ 
The $\alpha_{c}\left(L_{s}\right)$ and $\alpha_{i}\left(L_{s}\right)$ are drawn in Fig. 12a. The coating will crack if $\alpha_{c}\left(L_{s}\right)>\alpha_{i}\left(L_{s}\right)$. When $\alpha_{c}\left(L_{S}\right)<$ $\alpha_{i}\left(L_{s}\right)$, the coating will delaminate. As can be seen in Fig. 12a there is a transition point between cracking and delamination. The segment length where this transition happens is called the critical segment length $L_{c}$ given by $\alpha_{c}\left(L_{c}\right)=\alpha_{i}\left(L_{c}\right)$ :

$\frac{f_{1}}{F_{c}^{b}}\left(1-\operatorname{sech}\left(\frac{L_{s}}{2 \xi}\right)\right)=\frac{f_{2}}{F_{i}^{b}} \tanh \left(\frac{L_{s}}{2 \xi}\right)$

It follows that,

$L_{c}=2 \xi \ln \left(\frac{1+a}{1-a}\right) \quad$ with: $a=\frac{h_{i} l_{0} F_{c}^{b}}{\xi h_{c} F_{i}^{b}}$

and $0<a<1$. Equation 34 is plotted in Fig. 12b. The figure shows the influence of the correlation length $\xi$ on the interaction between cracking and delamination. During a simulation $\xi$ is a constant and $L_{S}$ decreases. So, a descending path along a vertical line will be followed in Fig. 12b. $L_{s}$ will decrease until it reaches $L_{c}$. Then the cracking will stop and the coating starts to delaminate. So, for $0<a<1$ the coating starts with cracking (if $L_{0}>L_{c}$ ) and eventually it will always delaminate. When $F_{c}^{b} / F_{i}^{b}$ decreases the $L_{c}$-curve in Fig. $12 \mathrm{~b}$ will move towards the axes and the area under the curve will be smaller and delamination starts at a smaller $L_{s}$ (or higher $\varepsilon$ ). When $a \geq 1$ the coating will only delaminate if $L_{0}<L_{c}$.

In this essentially scalar view, cracking and delamination alternate if disorder is introduced in the element failure properties. This is shown in Fig. 13a, where the interface has a disorder $\kappa_{i}=0.33$. This gives two additional curves for the interface elements, one with the minimum breaking strength $\left(\alpha_{i, \min }\right)$ and one with the maximum breaking strength $\left(\alpha_{i, \max }\right)$.

In the grey area delamination and cracking can interplay until $L_{s}$ is too small and breaking even the strongest interface elements is more favorable than cracking the coating. Figure $13 \mathrm{a}$ is converted into $13 \mathrm{~b}$ to show the influence of disorder in the interface. The thick curve is the $L_{c}$ calculated without disorder. The dashed lines are the upper and lower bounds for the case with disorder.

\subsubsection{Cracking and delamination in the discrete model}

The question is whether the simple conclusions drawn from the shear lag approximation also hold in the numerical simulations. In Fig. 14, the $L_{s}$ and the number of
Fig. 12 a $\alpha_{c}$ and $\alpha_{i}$ as function of $L_{s} . \xi=3.2$ (mm) and $a=0.35$ giving $L_{c}=4.6(\mathrm{~mm})$. b $L_{c}$ (black line) for $0<a<1$

Fig. $13 \alpha_{c}$ and $\alpha_{i}$ for $\kappa_{i} \neq 0$. In the grey area both cracking and delamination occur. $L_{c}$ for $\kappa_{i} \neq 0$
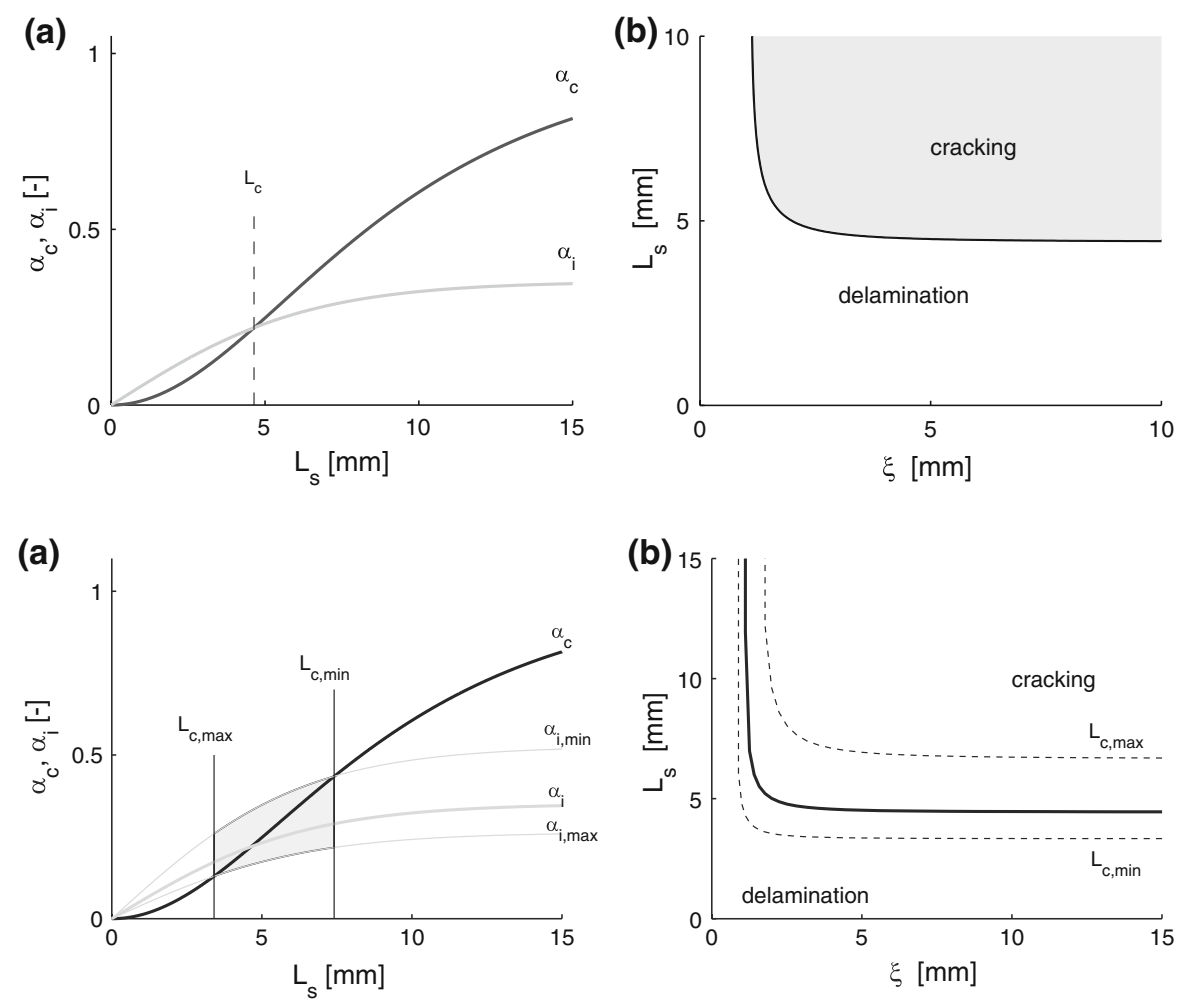
Fig. $14 L_{s}(\varepsilon)$ and $n_{d e l}(\varepsilon)$ for a simulation with delamination. $L_{s}$ and $n_{d e l}$ are both expressed in terms of number of springs

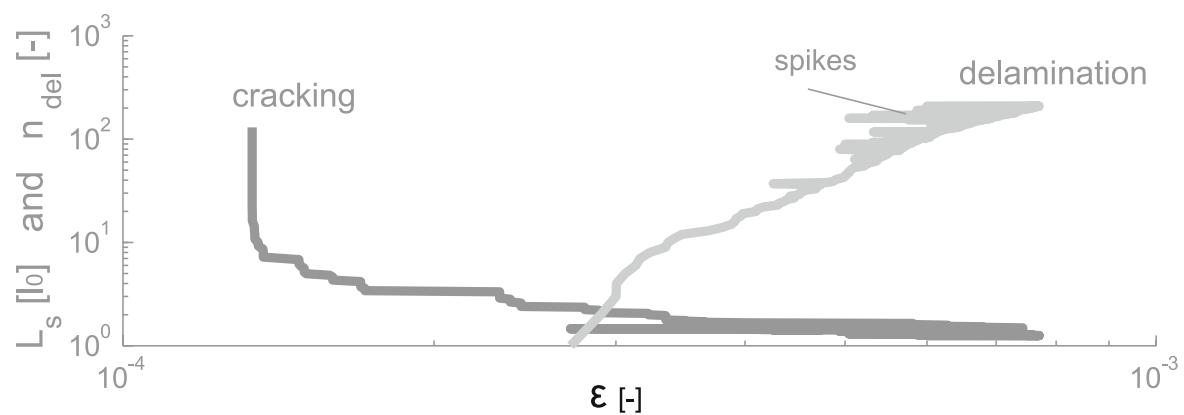

$\varepsilon[-]$

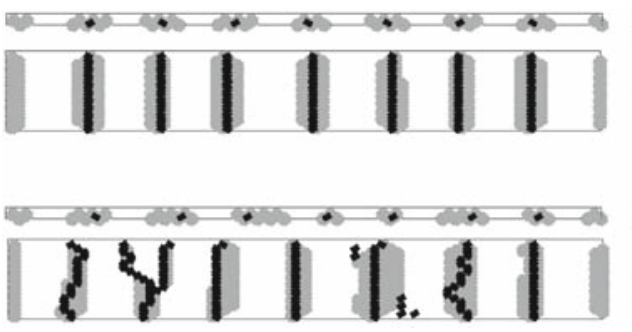

2D (a)

3D (b)

2D (c)

3D (d)
Fig. 15 Typical distribution of axial forces $F_{i}$ in the interface as function of the position $x L_{s}^{-1}$ in a segment. These forces are not included in the shear-lag models

delaminated elements, $n_{d e l}$ as a function of $\varepsilon$ are drawn for a numerical simulation with the same parameters as those used in Fig. 13a and b.

As evident from Fig. 13a the general picture derived from the shear lag approximation including disorder is valid. First cracking occurs, subsequently there is a regime of alternating cracking and delamination, and finally delamination takes over completely.

We note that because of the bending in the coating the stress distribution along the interface in general differs from that in the shear-lag approximation. Figure 15 shows a typical example. Quantitative discrepancies with the predictions of the shear-lag model may therefore perhaps be expected. It is expected that differences may also occur for delamination near corners or intersections of delamination fronts with free edges.

Figure 14 shows the number of delaminated interface springs vs. strain, revealing spikes, which are due to the simultaneous fracture of multiple interface elements. When an interface element situated at the edge of a segment fractures another interface element is now at the edge of the segment. Possibly, the new edge element is weaker than its predecessor and needs a lower $\varepsilon$ to fracture. Because of the solution method discussed
Fig. 16 Cracking (black) and delamination (grey) in 2D and 3D models. Sizes: $1 \times 91$ and $10 \times 91, L_{0} \xi^{-1}=17.2$. $2 \mathrm{D}$ after 50 broken elements and 3D after 400 broken elements. In the two upper simulations $\kappa_{c}=\kappa_{i}=\kappa_{g}=0$, in the two lower ones $\kappa_{c}=\kappa_{i}=0.125$

in chapter 2 the overall strain $\varepsilon$ is lowered to break this weaker element, causing the spikes.

In Fig. 14, information on where the delamination occurs with respect to the cracks is lacking. Results of simulations providing that spatial information are shown in Fig. 16, with $\kappa_{c}=\kappa_{i}=\kappa_{g}=0$ in Fig. 16a and b. Delamination is obviously located at the crack edges. In Fig. 16c-d similar simulations are shown except now with $\kappa_{c}=\kappa_{i}=0.125$ and $\kappa_{g}=0$. The cracks in the 3D model no longer propagate strictly perpendicular to the applied stress, but the delamination remains correlated with the crack edges. Even though the situation is less clear when there is disorder in the model, delamination still starts near cracks and prefers to initiate at the largest $L_{S}$.

\subsection{Bi-axial coating failure in 3D}

In bi-axially loaded 3D models, the first deformation mechanism is delamination at the corners. This leaves the remaining in-tact part of the of the interface with more rounded edges, after which cracking starts. This is shown in Fig. 17, where the interaction of cracking and 
Fig. 17 A bi-axially stretched $80 \times 813 \mathrm{D}$ model with delamination after 900 , 1800, 2700 and 3600 broken elements. $\kappa_{c}=0.25$, $\kappa_{i}=0.5$ and $\kappa_{g}=0.0$. $L_{0} \xi^{-1}=20.1$. Cracks (black) and delamination (grey)

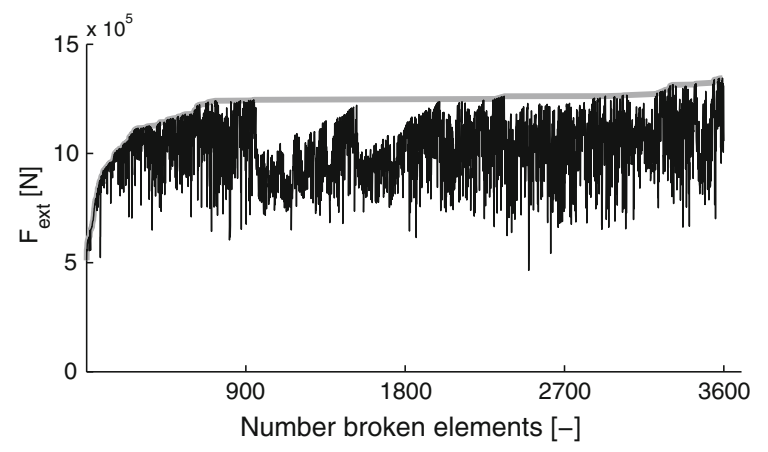

Fig. 18 The external force as a function of the number of broken elements for the simulation shown in Fig. 17. The gray line indicates the enveloping maximum force

delamination is shown at four stages. As can be seen in the first stage, there is only delamination at the edges of the model, after initiation form the corners. In the second stage, after 1800 broken elements, cracks have already appeared in the model. When the simulation is continued further, cracking is almost arrested but the delamination continues as shown in stages $3 \& 4$.

In displacement-controlled experiments a crack that has initiated may propagate without the need of increasing $F_{\text {ext }}$, reflecting a local snap-through behaviour. Evidently this is also captured in the simulations as shown in Fig. 18. After the first crack initiates, a much lower force is needed for propagation (see Sect.3.3, Fig. 10).

\subsubsection{Influence of disorder}

The influence of $\kappa_{i}$ in the interface is next investigated. The results are given in Fig. 19. The value of $\kappa_{i}$ changes from 0.0 to 1.0. The value of $F_{i}^{b}$ was changed to keep the "interface energy" $G$ of the interface the same in all three cases. We note that the interface energy (the energy needed to break all spring in the interface divided by the total interface area) scales with the second moment of the distribution of $F_{i}^{b}$. In case of a uniform distribution this amounts to
$G \propto F_{b}^{2}\left(1+\kappa_{i}^{2} / 3\right)$

The cases shown in Fig. 19 all have approximately the same G, but it can be seen that the relative amount of delamination and cracking may differ quite substantially.

Delamination and cracking patterns, as shown in Fig. 17, make it possible to define the mean delaminated area and mean segment area as a function of the macroscopic strain. Experimental results may be correlated to these quantities, giving insight in the amount of disorder in the coating and interface.

\section{Coating cracking, delamination and buckling}

In the lattice model the coating has a bending stiffness resulting from the spring network. It was verified that bending was captured in a realistic way using properties of the so-called Euler column the shape of which is given by Hutchinson and Suo (1992), Audoly (1999):

$y(z)=\frac{2 h_{c}}{\sqrt{3}} \frac{1+\cos \frac{2 \pi z}{l_{d}}}{2}\left(\frac{\sigma_{0}}{\sigma_{c}}-1\right)^{\frac{1}{2}}$

in which $\sigma_{0}$ is the external load and $\sigma_{c}$ is the critical external load when a buckle starts to form. $\sigma_{c}$ is defined as:

$\sigma_{c}=\frac{\pi^{2} E_{c} h_{c}^{2}}{3 l_{d}^{2}(1-v)}$

Controlled buckles were created using 2D models with pre-delaminated areas of fixed length, increasing the compressive stress and suppressing cracking in the coating. $\mathrm{y}(\mathrm{z})$ was measured as well as the relation between $\sigma_{c}$ and $h_{c}$ and $l_{d}$. It was found that the buckle shapes conformed closely to Eq. 36 and that $\sigma_{c}$ is proportional to $h_{c}^{2}$ and $l_{d}^{-2}$ as predicted by Eq. 37. In experiments it was found that buckles initiated in the scaling regime of cracking. More cracks would still appear for increasing strain while at the same time buckles initiated above delaminated areas along already existing cracks. This is in accordance with results in 
Fig. 19 Bi-axial $104 \times 121$

3D models with

delamination after 2000

broken elements.

$L_{0} \xi^{-1}=23.3, \kappa_{c}=0.25$

$\left(F_{c}^{b}=100 \pm 25(\mathrm{~N})\right)$ and

$\kappa_{g}=0.1$. Cracks (black)

and delamination (grey). a

$F_{i}^{b}=6 \pm 0(\mathrm{~N}), \mathbf{b}$

$F_{i}^{b}=4.5 \pm 1.5(\mathrm{~N}), \mathbf{c}$

$F_{i}^{b}=3 \pm 3(\mathrm{~N})$

Fig. 20 Three stages of a buckling process
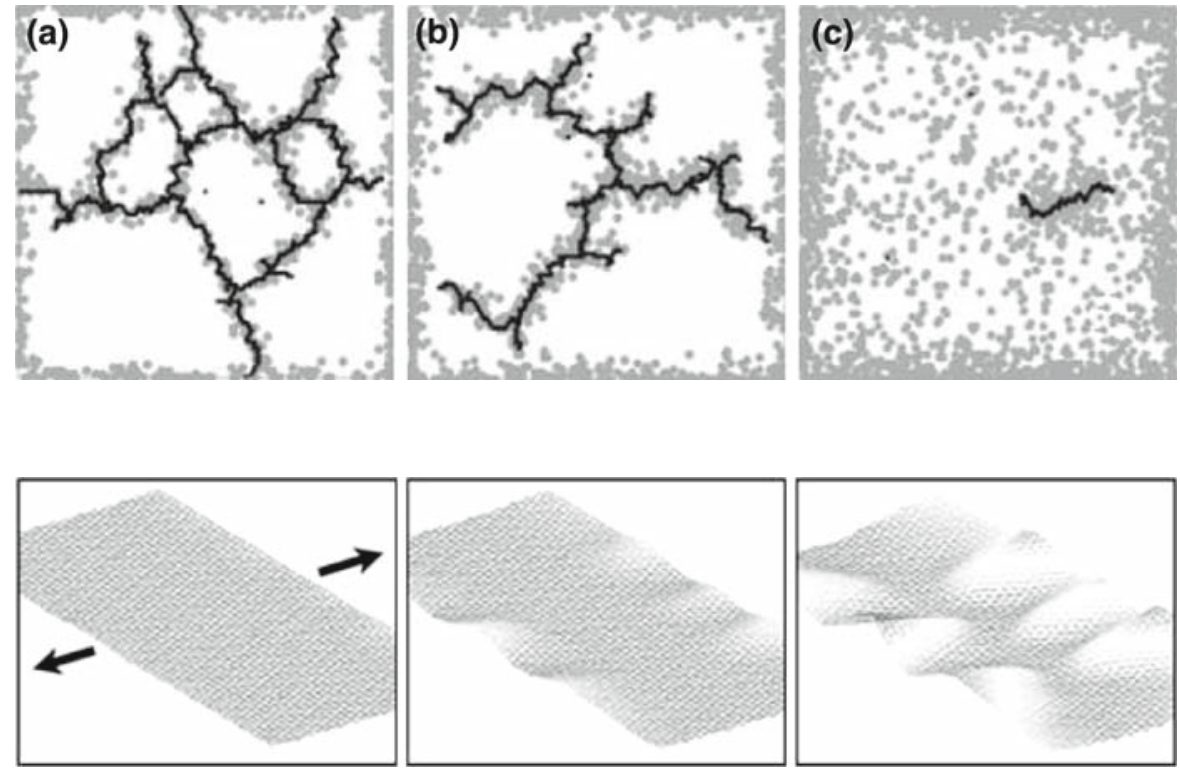

Weak interface springs act as initiation sites for the

Sect.4 where it was shown that cracking end delamination may occur at the same strain value for coatings and interfaces showing disorder. In this particular case disorder may be present in the coating, but plastic deformation of the Al substrate will certainly lead to roughening and to local differences in the interface energy release rate as well. Figure 1 shows a typical geometry of co-occurring cracking and buckling over delaminated areas. The buckles that form have a more or less triangular shape, widest near the crack face. As noted above and in Sect. 4 delamination will only become favorable above a certain crack density. To reduce the computational effort in the following we focus on an area between to already existing cracks. That is, we start from a model with a certain size and subsequently allow only for delamination and buckling. Initially all interface springs are intact, but their breaking strengths show disorder. Weak interface springs are therefore natural initiation points for delamination and buckling. In anticipation of the discussion later on it is noted that the interface area associated with a single broken spring and the energy released when it breaks are the only (implicit) limiting assumptions made on the nucleation of buckling in this description. Initially the model was subjected to a equi-biaxial compressive stress state and subsequently uniaxial strain was superimposed as indicated in Fig. 20. During the uniaxial tensile test the equi-biaxial compressive stress state changes to a stress state with tension along the tensile direction and compression perpendicular to it. buckles. When an area of the interface delaminates, the internal compressive stress causes the coating to bend upwards. Results of a typical calculation shown in Fig. 20, are indeed reproducing the experimentally observed behavior. Triangular buckles are clearly present near the edges of the mesh, which represent physical crack edges in this case.

\section{Discussion and conclusions}

A simple numerical model based on discrete linear elements has been proposed that describes cracking, delamination and buckling, and takes disorder and bending stiffness into account.

Some typical results concerning cracking, delamination and buckling are presented. The model has been shown to reproduce results from literature and experiments in a qualitative sense.

The advantages of choosing a lattice model to describe the interaction between cracking, delamination and buckling is that after definition of the geometry and breaking criteria no other assumptions are needed regarding nucleation, propagation and interaction of the dissipation mechanisms. However, as with every discrete description of physical phenomena it is useful to discuss to what extent the geometry of the model limits its applicability. Trivially, the choice of the spring lengths and strength determines the spatial resolution, 
the minimal crack opening, the minimal delaminated area and the minimal energy released in the model. Failure to represent relevant length-scales in the model can lead to misleading results. For instance it has been shown by Klein et al. (2001) in a study on cohesive zones that it may be necessary to spend a multiple of the interface energy $\mathrm{G}$ to delaminate an interface if it has been modeled by a grid that is too coarse. In cases were several dissipative phenomena may interact, such as cracking and delamination, "trapping" of one dissipation mode, say a crack, may lead to propagation in another mode, for instance delamination. This can potentially lead to qualitatively different behavior. It has been shown, in an apparently different context that the behavior of Si modeled by atomistic potentials may change from ductile to brittle depending on subtle changes in the potentials used (Bernstein and Hess 2003). In that case the anomalous presence of a ductile response (caused by dislocation emission at the crack tip) was associated with "lattice trapping" of a crack in the potential ahead of the crack tip that is corrugated on an atomic scale. Two relevant length scales were proposed, one for bond breaking and the other for elastic relaxation. The relative size of these length scales was found to be important. A bond-breaking length scale larger than the elastic relaxation length scale caused the breaking of a single bond to happen gradually as the crack advanced by several lattice periods, and lattice trapping was small. In the opposite case all of the bond-breaking energy was spent before elastic relaxation lowered the total energy, and lattice trapping was large. Such interplay between energy storing and dissipating phenomena clearly occurs in the model proposed here, the transition from cracking to delamination is a case in point. Here we have only shown that the model reproduces the co-occurrence of cracking and delamination observed in experiments. Whether and how exactly the transition from cracking to delamination is influenced by the model geometry is an open issue that deserves further study. Lengthscales associated with buckling are large compared to others length-scales involved (e.g. Hutchinson et al. 2000) and it is expected that if those are represented correctly, buckling will be faithfully reproduced provided the overall size of the model is large enough. With this in mind we turn to the following conclusions.

If limited to cracking only, the model reproduces $L_{s} \xi^{-1}\left(\varepsilon^{n}\right)$ curves for 2D cracking that are in agreement with the literature, and experiments. The interaction between disorder and cracking is also in agreement with the literature.

An essentially scalar (shear lag) representation of the model indicates the existence of a transition point (i.e. critical segment length) $L_{c}$ between cracking and delamination for $\kappa_{c}=\kappa_{i}=0$ and a well-defined, range of segment lengths at which cracking and delamination occur side-by-side in case of disordered coatings. This behavior is also retrieved by the model.

A comparison between 2D and 3D uni-axial simulations with delamination is made to verify that $L_{c}$ is also present in 3D models. Uni-axial simulations without disorder give the same results for both $2 \mathrm{D}$ and $3 \mathrm{D}$ models. When the disorder is increased, differences between 3D and 2D simulations appear due to the crack propagation.

The main distinguishing characteristic of the proposed model, a 3D yet simple network of elements in the coating that provides a bending stiffness, reflects a qualitatively correct interaction between delamination and buckling behavior. Specifically, the experimentally observed initiation of triangular buckles above delaminated areas on crack edges is faithfully reproduced.

Acknowledgements This work was financed by SENTER/IOP under grant IOT97002.

Open Access This article is distributed under the terms of the Creative Commons Attribution Noncommercial License which permits any noncommercial use, distribution, and reproduction in any medium, provided the original author(s) and source are credited.

\section{References}

Alava MJ, Nukala PKKV, Zapperi S (2006) Statistical models of fracture. Adv Phys 55:349-476

Audoly B (1999) Stability of straight delamination blisters. Phys Rev Lett 83(20):4124-4127

Bernstein N, Hess DW (2003) Lattice trapping barriers to brittle fracture. Phys Rev Lett 91:025501

Beuth JL (1992) Cracking of thin bonded films in residual tension. Int J Solids Struct 29(13):1657-1675

Gille G (1984) Investigations on mechanical behaviour of brittle wear-resistant coatings, II: theory. Thin Solid Films 111:201-218

Gille G, Wetzig K (1983) Investigations on mechanical behaviour of brittle wear-resistant coatings, I: experimental results. Thin Solid Films 110:37-54

Handge UA, Sokolov IM, Blumen A (1997) Fragmentation of viscoelastic surface layers. Europhys Lett 40(3):275-280

Handge UA, Leterrier Y, Manson JAE, Sokolov IM, Blumen A (1999) An analysis of disorder in thin silicon oxide coatings. Europhys Lett 48(3):280-285 
Handge UA, Sokolov IM, Blumen A (2000a) Universal scaling and nonlinearity in surface layer fragmentation. Phys Rev E 61(3):3216-3219

Handge UA, Leterrier Y, Sokolov IM, Blumen A (2000b) Two scaling domains in mutiple cracking phenomena. Phys Rev E 62(6):7807-7810

Handge UA, Sokolov IM, Blumen A (2001) Disorder and plasticity in the fragmentation of coatings. Phys Rev E 64:016109

He MY, Evans AG, Hutchinson JW (1998) Effects of morphology on the decohesion of compressed thin films. Mat Sci Eng A 245:5168-181

Hornig T, Sokolov IM, Blumen A (1996) Patterns and scaling in surface fragmentation processes. Phys Rev E 54(4):42934298

Hutchinson JW, Suo Z (1992) Mixed mode cracking in layered materials. Adv Appl Mech 29:63-191

Hutchinson JW, He MY, Evans AG (2000) The influence of imperfections on the nucleation and propagation of buckling driven delaminations homogenization scheme. J Mech Phys Solids 48:709-734

Jagla EA (2007) Modeling the buckling and delamination of thin films. Phys Rev B 75:085405

Klein PA, Foulk JW, Chen EP, Wimmer SA, Gao HJ (2001) Physics-based modeling of brittle fracture: cohesive formulations and the application of meshfree methods. Theor Appl Fract Mech 37:99-166

Kitsuzenaki S (1999) Fracture patterns induced by desiccation in a thin layer. Phys Rev E 60(6):6449-6464

Kouznetsova V, Geers MGD, Brekelmans WAM (2002) Multiscale constitutive modelling of heterogeneous materials with a gradient-enhanced computational homogenization scheme. Int J Numer Meth Eng 54:1235-1260

Leung K-t, Neda Z (2000) Pattern formation and selection in quasistatic fracture. Phys Rev Lett 85(3):662-666
Leung K-t, Josza L, Ravasz M, Neda Z (2001) Spiral cracks without twisting. Nature 410:166

Meakin P (1987) A simple model for elastic fracture in thin films. Thin Solid Films 151:165-190

Mezin A, Lepage J, Pacia N, Paulmier D (1989a) Étude statistique de la fissuration de revêtements. I: théorie. Thin Solid Films 172:179-209

Mezin A, Pacia N, Nivoit M, Weber B (1989b) Étude statistique de la fissuration de revêtements. I: théorie. Thin Solid Films 172:211-225

Morgenstern O, Sokolov IM, Blumen A (1993) Analysis of a one-dimensional fracture model. J Phys A 26:4521-4537

Ostoja-Starzewski M (2002) Lattice models in micromechanics. Appl Mech Rev 55:35-60

Skjeltorp AT, Meakin P (1988) Fracture in microsphere monolayers studied by experiment and computer simulation. Nature 335:424-426

Mier JGMvan (2007) Multi-scale interaction potentials (F-r) for describing britle disordered materials like cement and concrete. Int J Fract 143:41-78

Walmann T, Malthe-Sorenssen A, Feder J, Jossang T, Meakin P (1996) Scaling relations between lengths and widths of fractures. Phys Rev Lett 77(27):5393-5397

Xia CZ, Hutchinson JW (2000) Crack patterns in thin films. J Mech Phys Solids 48:1107-1131

Yanaka M, Tsukahara IM, Y, Nakaso N (1998) Cracking phenomena of brittle films in nanostructure composites analysed by a modified shear lag model with residual strain. J Mater Sci 33:2111-2119

Yanaka M, Kato Y, Tsukahara IM, Y, Takeda N (1999) Effects of temperature on the multiple cracking progress of submicron thick glass films deposited on a polymer substrate. Thin Solid Films 355-356:337-342, 268 